\section{DUAL-BAND FEEDFORWARD LINEAR POWER AMPLIFIER FOR DIGITAL CELLULAR AND IMT-2000 BASE- STATION}

\author{
Heungjae Choi, ${ }^{1}$ Yongchae Jeong, ${ }^{1}$ J. S. Kenney, ${ }^{2}$ and \\ Chul Dong Kim ${ }^{3}$ \\ ${ }^{1}$ Department of Electronics and Information Engineering, Chonbuk \\ National University, Jeonju, Republic of Korea; Corresponding author: \\ streetpoet@chonbuk.ac.kr \\ 2 School of ECE, Georgia Institute of Technology, Atlanta, GA \\ ${ }^{3}$ Sewon Teletech, Inc., Anyang, Kyunggi, Republic of Korea
}

\section{Received 27 July 2008}

ABSTRACT: This article presents the design of a novel dual-band signal cancellation circuit and its application to feedforward linear power amplifier. Intermodulation distortion cancellation loop shows $20.45 d B$ and $25.04 d B$ suppression for digital cellular band and IMT-2000 band, respectively, over a correction bandwidth of 100 MHz. From the adjacent channel leakage ratio measurement, 16.5 $d B$ and $18.6 d B$ improvements are achieved for each band under simultaneous operation. () 2009 Wiley Periodicals, Inc. Microwave Opt Technol Lett 51: 922-926, 2009; Published online in Wiley InterScience (www.interscience.wiley.com). DOI 10.1002/mop.24198

Key words: dual-band; feedforward; power amplifier

\section{INTRODUCTION}

A few decades ago, mobile communication was limited to low data rate signals exchange such as voice or text data. However, higher data rate signal exchange has become essential part of mobile communication since several additional applications such as mega pixel digital cameras, $\mathrm{mp} 3$ players, and digital TV receiv-

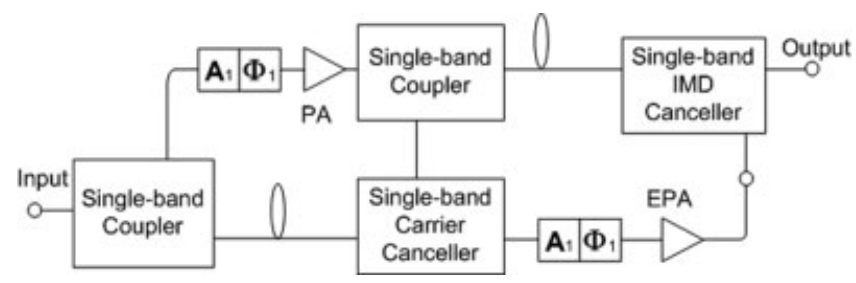

Figure 1 Conventional single-band FFW LPA ers are integrated into the mobile unit to satisfy various demands from the users.

To provide additional services including multimedia data, a service provider has to utilize more than one frequency band. A new service requires a considerable investment in research and development for each application. For that reason, active studies are being done on broadband, multiband devices that can cover more than one frequency band under simultaneous operation. However, due to the rigid linearity requirements of the base-station power amplifier, the research has been limited to the mobile station power amplifier [1]. This article addresses the design of simultaneous multiband base-station linear power amplifiers (LPAs). Feed-forward (FFW) is one of the most widely used techniques with excellent linearity improvement [2, 3]. However, previous works were mainly focused on the extension of operation bandwidth and on the efficiency improvement in one band of operation [4-6].

We propose the design of equal group delay dual-band signal cancellation circuits to construct a dual-band FFW LPA. To provide the experimental verification, we implemented the dual-band FFW LPA, and showed the loop cancellation and adjacent channel leakage ratio (ACLR) improvements at both bands under simultaneous operation.

\section{DUAL-BAND FFW LPA}

Figure 1 shows a conventional single band FFW LPA, and Figure 2 shows the block diagram of the proposed dual-band FFW LPA. Circuit components in the conventional FFW LPA amplify, divide, and combine the signals in just one band of operation. The dualband FFW LPA consists of a broadband Wilkinson power divider, input and output diplexers, a broadband directional coupler, a dual-band carrier cancellation circuit, and a dual-band IMD cancellation circuit. Variable attenuators, phase shifters, main power amplifiers (PAs), and error power amplifiers (EPAs) are provided for each band of operation.

The proposed dual-band FFW LPA has the same structure as the conventional one, with the major difference being that the PA and EPA are replaced by the dual-band PA and EPA using diplexers. One of major design issues in LPA is high efficiency. Because high power transistor has very low resistance for the optimum in/output power matching, the simultaneous dual-band matching with just one transistor is very difficult. Also its power capacity and efficiency are decreased drastically. Instead, the separated

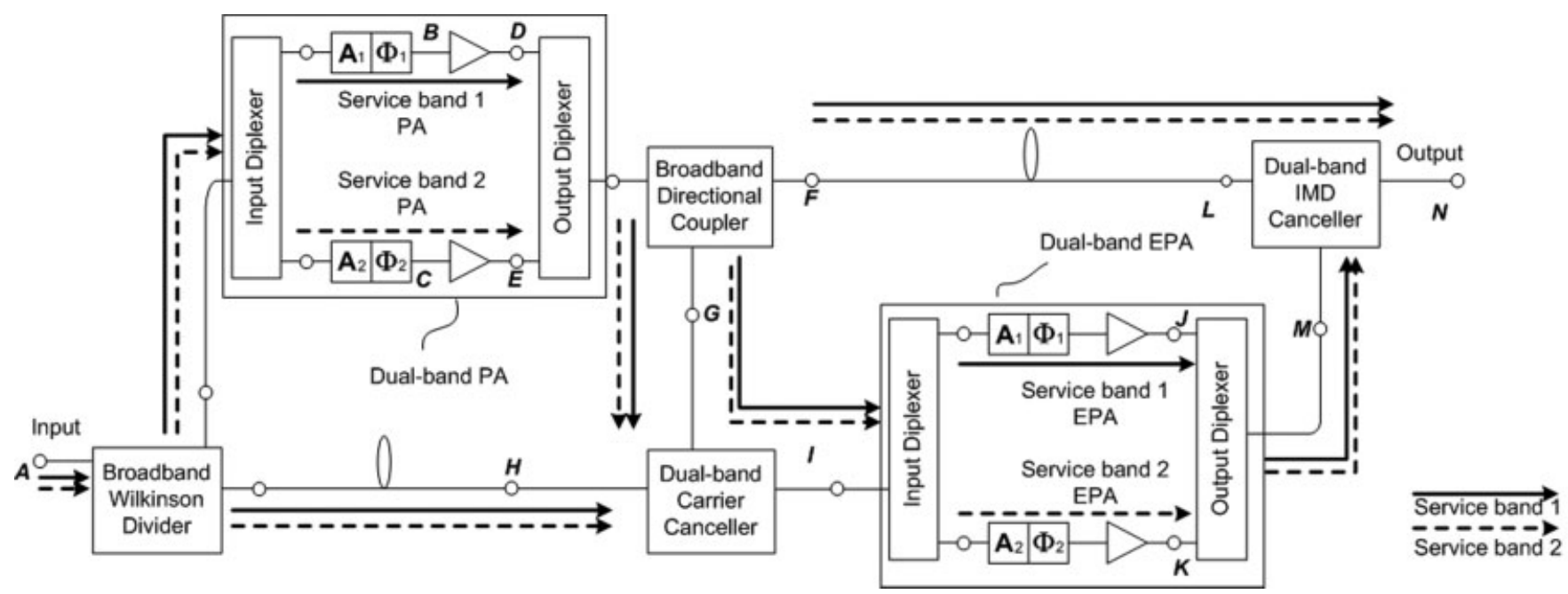

Figure 2 Block diagram of the proposed dual-band FFW LPA 


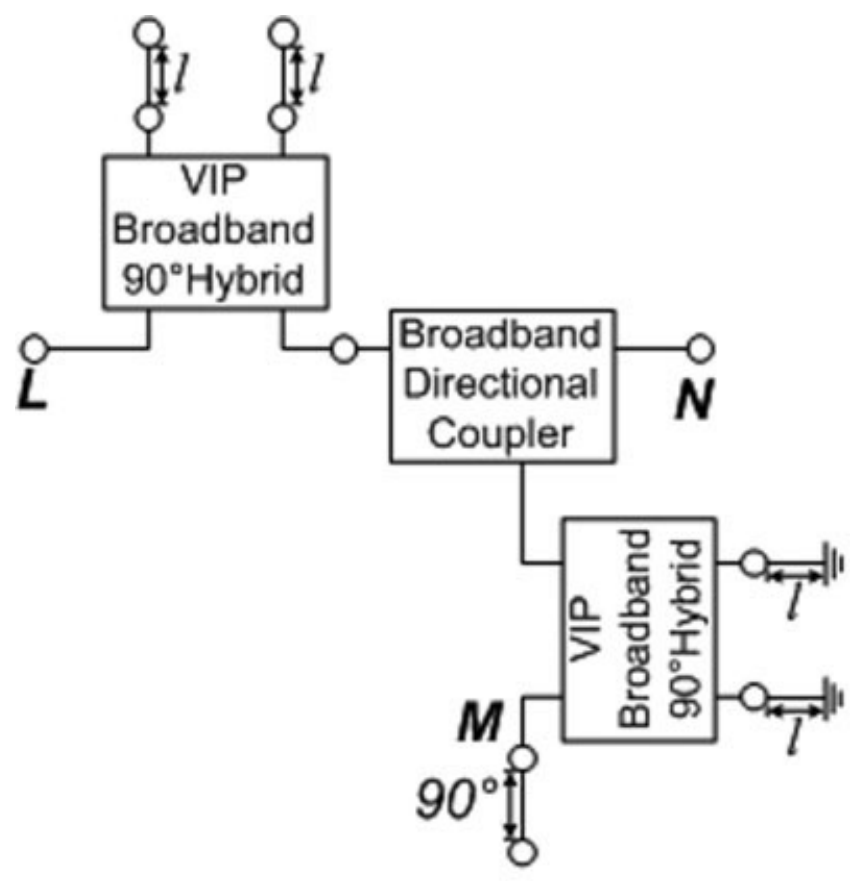

(a)

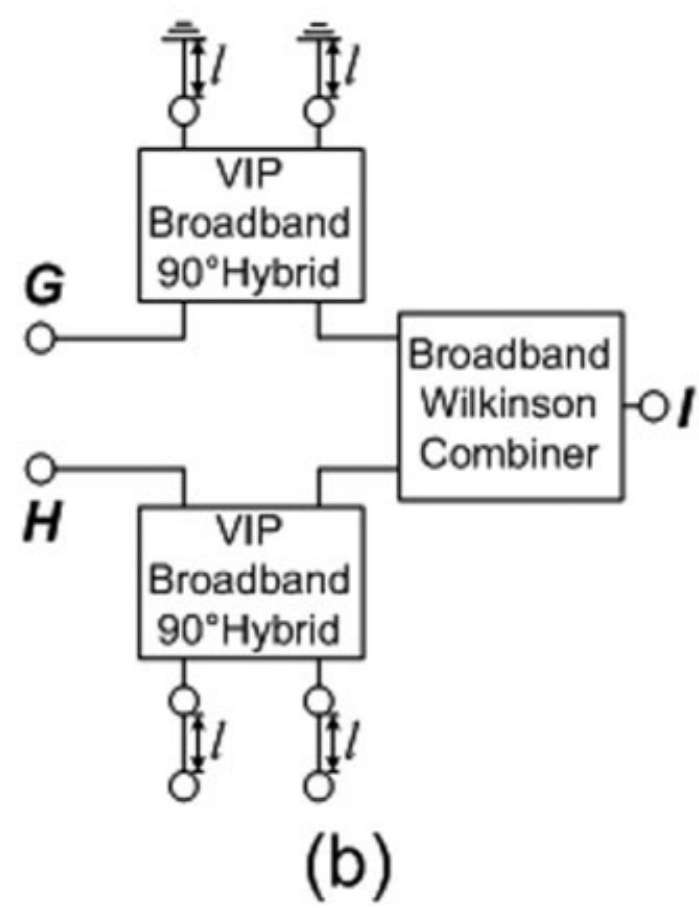

Figure 3 Block diagram of the dual-band signal cancellation circuit: (a) Carrier canceller and (b) IMD canceller

dual-band PAs are more effective to obtain high power and efficiency. High power diplexers must be used at the output of the PAs and EPAs. However, the other diplexers can be easily implemented with low Q components because the signal level is low at the input. Also, dual-band signal cancellation circuits replace single band couplers and single band signal cancellers.

The principle of operation of the dual-band FFW LPA is similar to the conventional single-band FFW LPA except for the dual-band PA and the dual-band signal cancellation circuit. If we denote the two band of operation as service band 1 (solid line) and service band 2 (dotted line) in Figure 2, section with both lines means that there are dual-band signals, and section with only one line presents that there is a single band signal through the frequency selective characteristic of the diplexer.

When designing the dual-band PA, the isolation characteristic of a diplexer should be carefully considered because the proposed configuration is designed for the high power application [7]. In this article, the diplexer has an isolation of $45 \mathrm{~dB}$ rejection for digital cellular band and $35 \mathrm{~dB}$ rejection for IMT-2000 band. This result is enough not to introduce any interference to the other band of operation for $120 \mathrm{~W}$ PEP base-station power amplifiers designed in the conventional manner.

\section{DUAL-BAND SIGNAL CANCELLATION CIRCUIT WITH BROAD BANDWIDTH}

To obtain broadband signal cancellation, broadband amplitude, phase, and group delay matching are essential, and must be matched at each band simultaneously. Because of the fact that the conventional signal canceller cannot satisfy the out-of-phase and equal group delay matching at the same time inherently, an equal group delay signal canceller has been proposed [6]. The principle of the equal group delay signal canceller is that the reflection coefficients of transmission lines of same length, $l$, that are terminated with short and open condition, respectively, have out-ofphase and equal group delay characteristic at the same time. Figure 3 shows the block diagram of dual-band signal canceller using the equal group delay. Dual-band carrier cancellation circuit consists of broadband Wilkinson combiner and broadband $90^{\circ}$ hybrids [8]. Dual-band IMD cancellation circuit is composed of broadband directional coupler and broadband $90^{\circ}$ hybrids.

When the dual-band signals are applied to the two input ports $(\mathrm{G}, \mathrm{H})$ of the dual-band carrier canceller in Figure 3(a), signals of each band are fed to broadband $90^{\circ}$ hybrid of which the coupling and the through port are terminated with open and short condition, respectively. The two output signals of the broadband $90^{\circ}$ hybrid are out-of-phase, and fed into in-phase broadband Wilkinson combiner. Because the two input signals at the final output port (I) experience the same group delay time and are out-of-phase, nearly perfect signal cancellation with broad bandwidth can be achieved for dual-band operation. The amplitude matching is achieved by the variable attenuators in front of the PAs. The broadband $90^{\circ}$ hybrid is used to obtain good reflection characteristic, and it can be replaced with small size commercial broadband $90^{\circ}$ hybrid component for size reduction. The operating principle of the dual-band IMD cancellation circuit in Figure 3(b) is almost same except $90^{\circ}$ phase compensation of a loose coupling broadband directional coupler. The matching of magnitude between two paths is obtained with variable attenuators in front of the EPA. Figure 4 is the photograph of the fabricated carrier cancellation circuit.

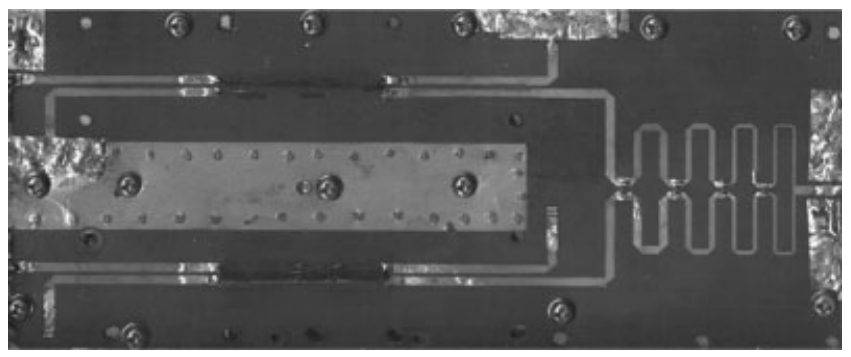

Figure 4 The photograph of the fabricated dual-band carrier cancellation circuit 
Figure 5 shows the measured phase balance of the dual band carrier cancellation circuit between two paths at each operation bands. Phase flatness at digital cellular band $(869-894 \mathrm{MHz})$ and IMT-2000 band $(2110-2170 \mathrm{MHz})$ are $\pm 0.7^{\circ}$ and $\pm 0.5^{\circ}$, respectively. This phase variation is thought to be induced by the fact that the center frequency of the broadband $3 \mathrm{~dB}$ hybrid is set at the arithmetic mean value $(1510 \mathrm{MHz})$ of $880 \mathrm{MHz}$ and $2140 \mathrm{MHz}$, causing a phase error from $90^{\circ}$ at the center frequencies of each band of operation. Although the phase variation is negligible in this case, this phenomenon may limit the cancellation bandwidth of the dual-band signal cancellation circuit.

\section{EXPERIMENTAL RESULTS AND DISCUSSIONS}

To prove the validity of the proposed configuration, we implemented dual-band FFW LPA for digital cellular and IMT-2000 band, which are separated about $1.26 \mathrm{GHz}$ between center frequencies, and measured the performance of the implemented linearization system with the commercial power amplifier of $120 \mathrm{~W}$ PEP for base-station.

Figure 6(a) shows the 1st loop signal cancellation result using the proposed dual-band carrier canceller measured with a network
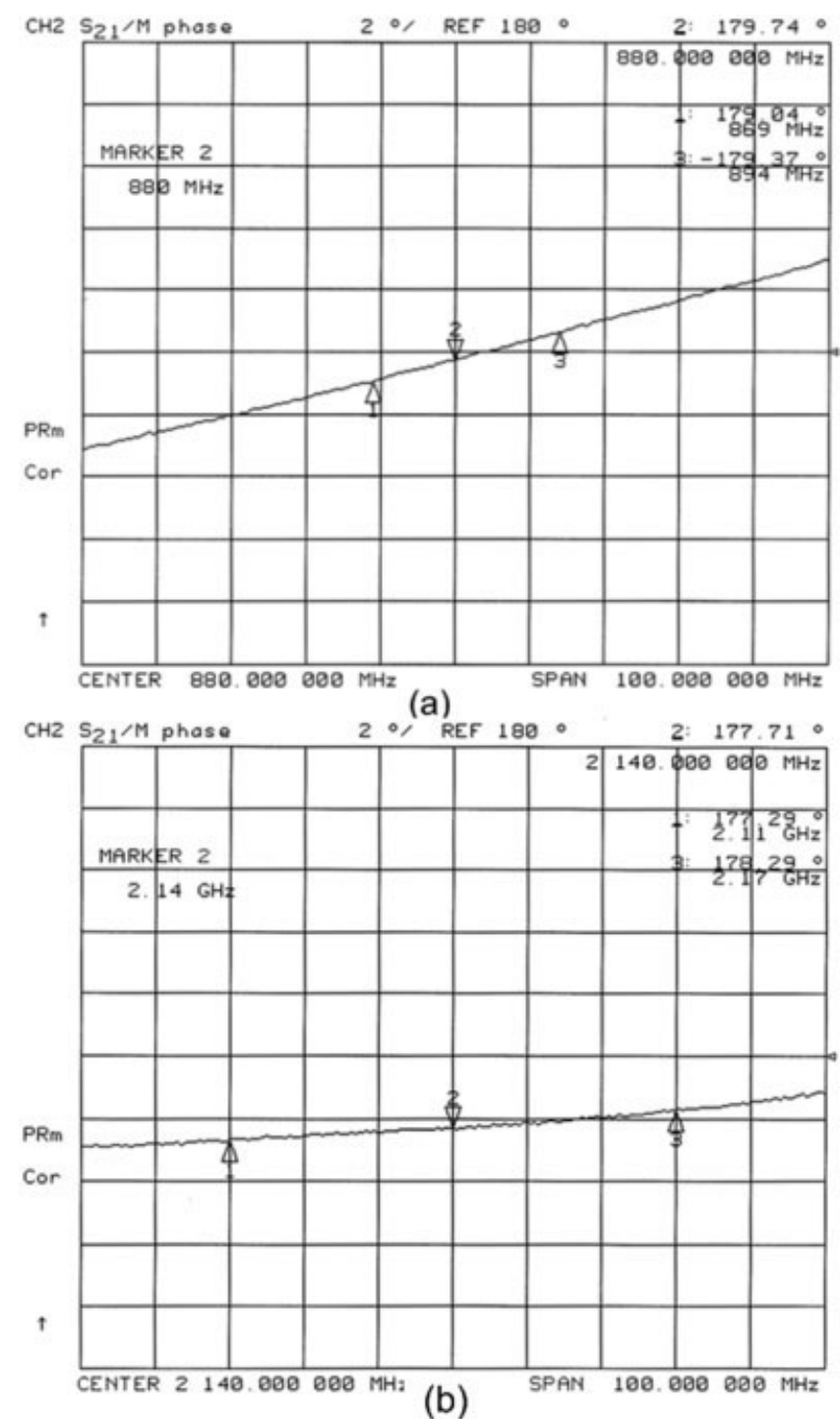

Figure 5 Measured phase flatness of the dual-band carrier canceller at: (a) digital cellular band and (b) IMT-2000 band

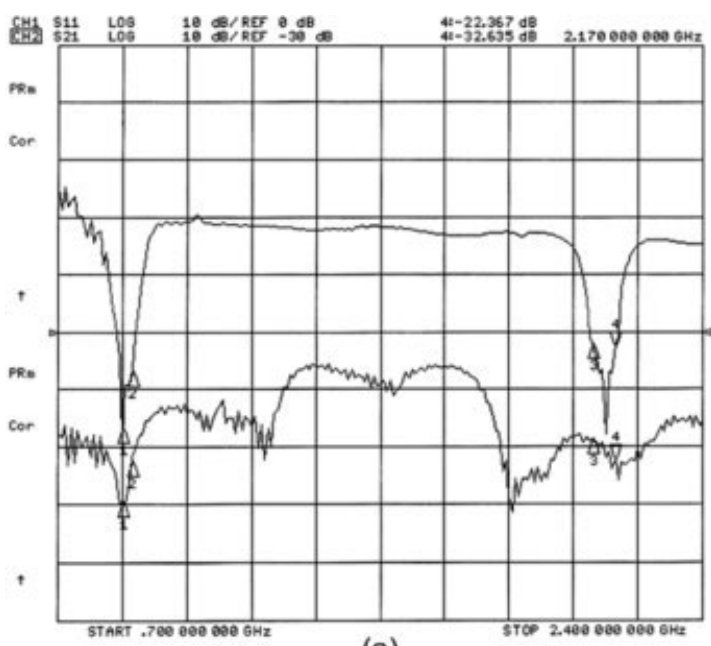

CH1 Markeri 15.29.619.8B

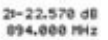

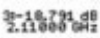

(a)

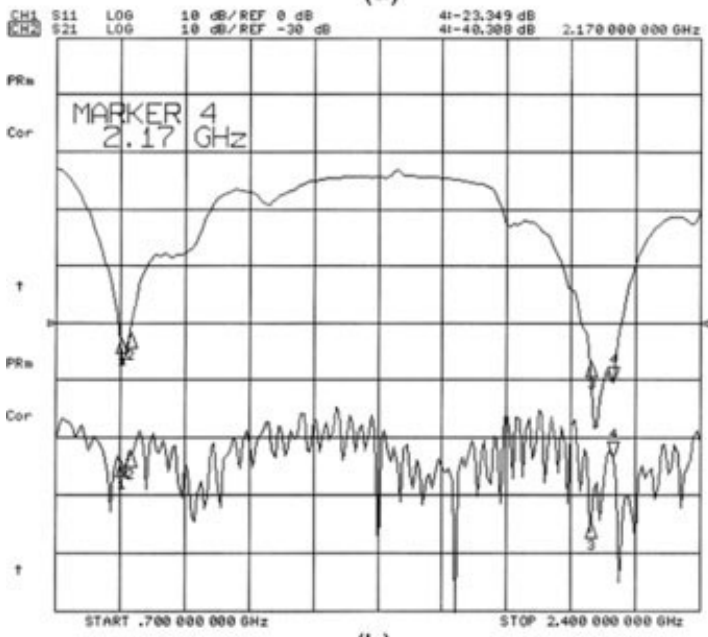

CH1 Marker

13-24.355 21-22.570 of: 2.114.385:

(b)

Figure 6 Measured dual-band (a) Carrier cancellation loop and (b) IMD cancellation loop

analyzer. The proposed canceller cancels the input signal more than $36.9 \mathrm{~dB}$ for digital cellular band and $32.1 \mathrm{~dB}$ for IMT-2000 band, simultaneously. Figure 6(b) just shows the IMD signal cancellation characteristic using the proposed dual-band IMD canceller. The input signal is cancelled more than $21.8 \mathrm{~dB}$ within digital cellular band and more than $30.7 \mathrm{~dB}$ within IMT-2000 band. The frequency bandwidth that the signal is cancelled more than $20 \mathrm{~dB}$ is over $113.27 \mathrm{MHz}$ and $173.85 \mathrm{MHz}$ for digital cellular and IMT-2000 band, respectively.

For the experimental verification, we have measured the output power spectral density of the dual-band FFW LPA with and without FFW loop using a forward-link CDMA IS-95A 4-carrier signal for digital cellular band and WCDMA 4-carrier signal for IMT-2000 band at the same time. These measurement results are shown in Figures 7 and 8, respectively. ACLRs at 3.125 MHz and 4.375 $\mathrm{MHz}$ offset are shown through output dynamic range at digital cellular band in Figure 7(a). Figure 7(b) shows the measured power spectral density of the implemented dual-band FFW LPA for digital cellular band at an average output power of 41.5 $\mathrm{dBm}$ before and after cancellation. The ACLR at 3.125 MHz offset is $-49.5 \mathrm{dBc}$, improved about $16.5 \mathrm{~dB}$ by the cancellation. The amount of improvement is smaller than expected from the result of network analyzer because of the limitation of the measurement setup. The proposed system shows excellent linearity through output dynamic range. 
ACLRs at $5 \mathrm{MHz}$ and $10 \mathrm{MHz}$ offset are shown through output dynamic range at IMT-2000 band in Figure 8(a). Figure 8(b) shows the measured power spectral density of the implemented dual-band FFW LPA for IMT-2000 band at an average output power of $40 \mathrm{dBm}$ before and after cancellation. The ACLR at 5 $\mathrm{MHz}$ offset is $-51.7 \mathrm{dBc}$, improved about $18.6 \mathrm{~dB}$ by the cancellation. The proposed system also shows excellent linearity through output dynamic range at IMT-2000 band.

\section{CONCLUSION}

For simultaneous linear amplification of a two frequency band signal, we proposed the design technique of using a dual-band equal group delay carrier canceller, a dual-band equal group delay IMD canceller, and a dual-band FFW LPA as an extension of the single-band FFW technique.

The proposed design method of the dual-band FFW LPA can be extended to operate at center frequency separated by more than $1 \mathrm{GHz}$, with the bandwidth of diplexer and broadband directional coupler being the limiting factors. Moreover, due to the advantage of the equal group delay signal canceller, broadband operation may be extended for more than two bands of operation. Therefore, it is

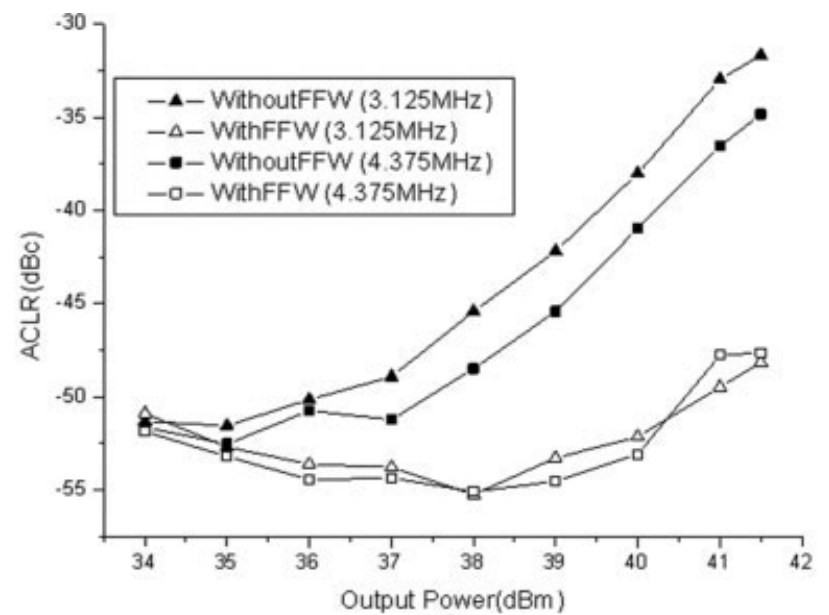

(a)

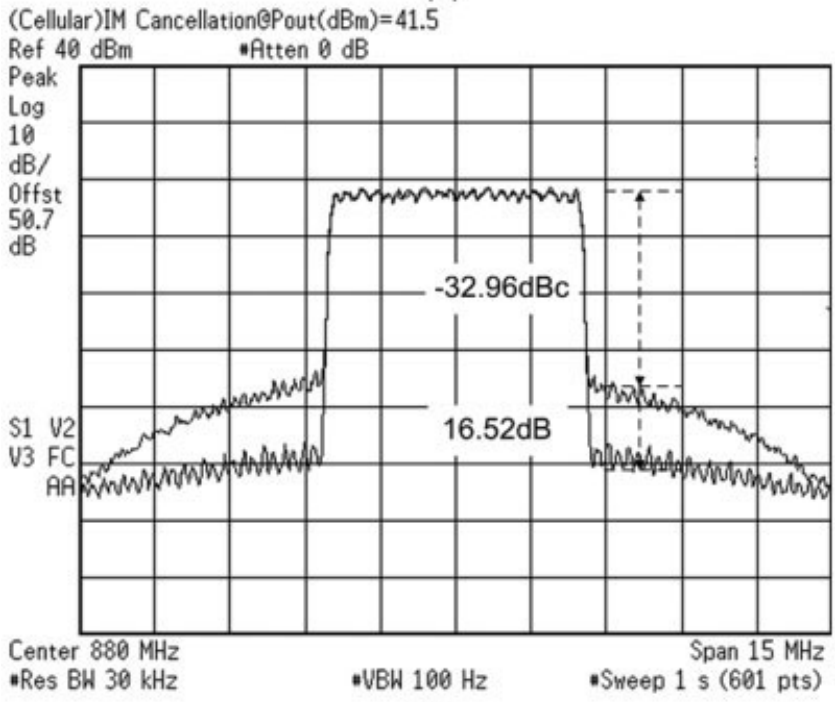

(b)

Figure 7 Measured ACLRs and power spectral density of the dual-band FFW LPA with and without FFW loop at digital cellular band: (a) ACLR characteristics through the output dynamic range and (b) Power spectral density at an average output power of $41.5 \mathrm{dBm}$

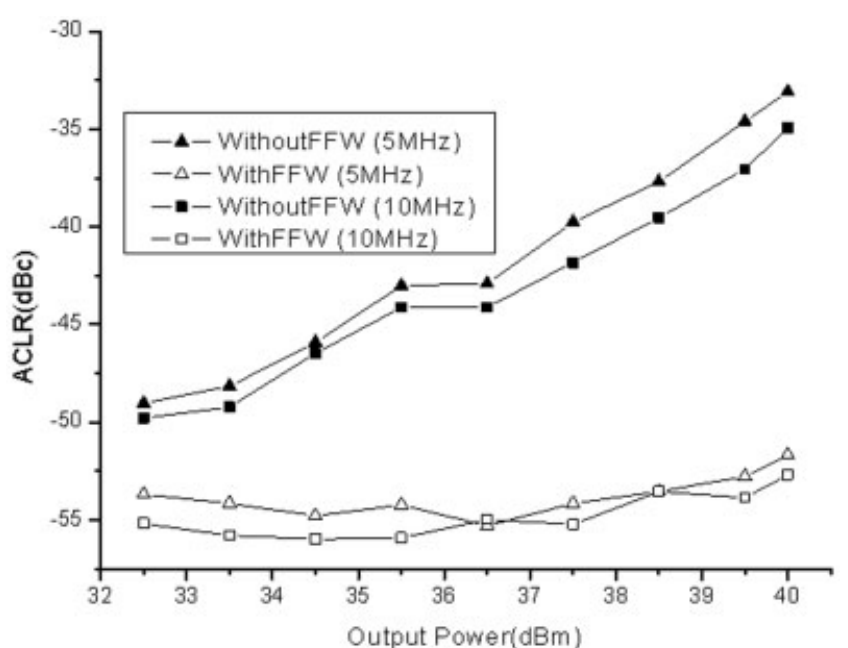

(a)

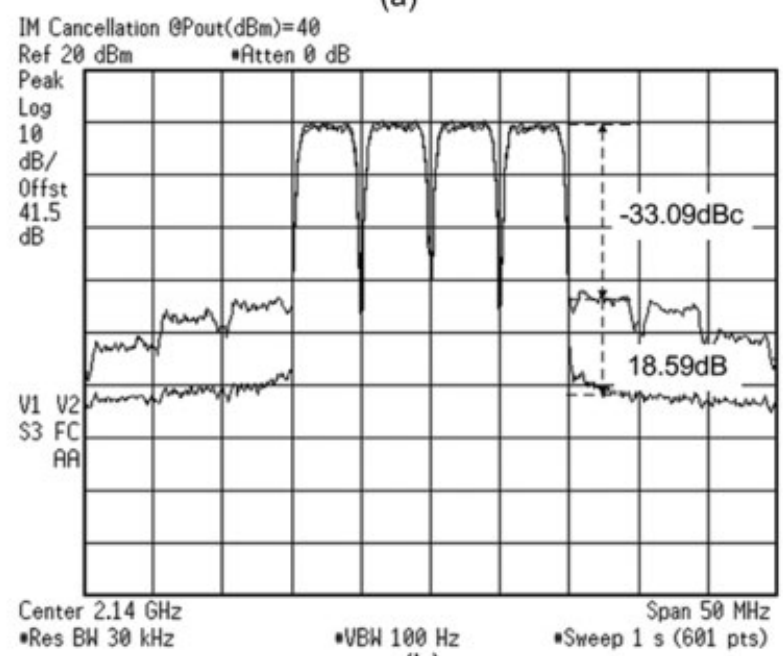

(b)

Figure 8 Measured ACLRs and power spectral density of the dual-band FFW LPA with and without FFW loop at IMT-2000 band: (a) ACLR characteristics through the output dynamic range and (b) power spectral density at an average output power of $40 \mathrm{dBm}$

expected that the proposed system would show its superior ability as a multiband base-station linear power amplifier in the broadband multimedia communication environment. There are several advantages to this proposed architecture, such as reduction of the number of antennas, high power coaxial cables, and other additional elements of the RF system.

\section{ACKNOWLEDGMENTS}

This work is partially supported by the second stage of Brain Korea 21 Project.

\section{REFERENCES}

1. K. Kunihiro, S. Yamanouchi, T. Miyazaki, Y. Aoki, K. Ikuina, T. Ohtsuka, and H. Hida, A diplexer- matching dual-band power amplifier LTCC module for IEEE $802.11 \mathrm{a} / \mathrm{b} / \mathrm{g}$ wireless LANs, IEEE Radio Frequency Integrated Circuits Symposium, Fort Worth, TX, 2004, pp. 303-306.

2. S.C. Cripps, Advanced techniques in RF power amplifiers design, Artech House, Norwood, MA, 2002.

3. F.H. Raab, P. Asbeck, S.C. Cripps, P.B. Kenington, Z.B. Popović, V. Pothecary, J.F. Sevic, and N.O. Sokal, Power amplifiers and transmitters for RF and Microwave, IEEE Trans Microwave Theory Tech 50 (2002), 814-826. 
4. Y.-C. Jeong, Y.-J. Song, I.-J. Oh, and C.-D. Kim, A novel adaptive feedforward amplifier using an analog controller, Microwave J 46 (2003), 76-85.

5. Y.-C. Jeong, A feedforward power amplifier with loops to reduce RX band noise and intermodulation distortion, Microwave J 45 (2002), 80-91.

6. Y.-C. Jeong, D. Ahn, C.-D. Kim, and I.-S. Chang, Feedforward amplifier using equal group-delay signal canceller, IEEE International Microwave Symposium, San Francisco, CA, 2006, pp. 1530-1533.

7. H.-J. Choi, H.-Y. Jeong, Y. Kim, J.-S. Lim, C.-D. Kim, and Y.-C. Jeong, Dual-band predistortion power amplifier for digital cellular and IMT-2000 base-station application, Asia-Pac Microwave Conference 2, Suzhou, China, 2005, pp. 208-211.

8. I.S. Kim and C.S. Lee, A study on broadband hybrid design using vertically installed planar circuit with partially removed ground plane, J KEES 16 (2005), 661-670.

(C) 2009 Wiley Periodicals, Inc.

\section{MINIATURIZATION OF NARROW-BAND POWER DIVIDERS BY USING CPW METAMATERIAL TRANSMISSION LINES}

\author{
Francisco Aznar, Jordi Bonache, Alexandre Valcarcel, and \\ Ferran Martín \\ GEMMA/CIMITEC, Departament d'Enginyeria Electrònica, Universitat \\ Autònoma de Barcelona, 08193 BELLATERRA (Barcelona), Spain; \\ Corresponding author: Ferran.Martin@uab.es
}

Received 31 July 2008

ABSTRACT: In this article, it is demonstrated that the size of planar microwave circuits and components can be reduced by using coplanar waveguide $(C P W)$ metamaterial transmission lines. The small size and the possibility to control the electrical characteristics (phase and characteristic impedance) of these lines, make them suitable for compact planar device design. To illustrate the possibilities of the approach, different power dividers, consisting on split-ring resonators (SRRs) and spiral resonators (SRs) loaded CPWs, have been designed, fabricated (in a conventional low loss microwave substrate) and measured. Sixty percentage size reduction, as compared to conventional devices, has been achieved. This size reduction can be enhanced by using smaller resonators based on different topologies. (C) 2009 Wiley Periodicals, Inc. Microwave Opt Technol Lett 51: 926-929, 2009; Published online in Wiley InterScience (www.interscience.wiley.com). DOI 10.1002/mop. 24195

Key words: metamaterials; split-ring resonator (SRRs); transmission lines; power dividers

\section{INTRODUCTION}

Many techniques devoted to size reduction in planar microwave circuits and components have been proposed in the literature. This is due to the fact that planar microwave components usually exhibit large dimensions, and this can be a critical aspect in certain applications. One of these approaches consists on the implementation of microwave components by means of metamaterial transmission lines. Such lines are artificial lines consisting on a host line loaded with reactive elements, and they can be implemented by means of two main approaches: (i) the CL-loaded approach, where conventional transmission lines are loaded with series capacitances and shunt inductances [1-3] and (ii) the resonant-type approach, where the lines are loaded with sub-wavelength resonators, such as split-ring resonators (SRRs) or complementary split-ring resonators (CSRRs) combined with shunt inductances and series capac- itances, respectively $[4,5]$. These lines exhibit controllable electrical characteristics (phase and characteristic impedance), beyond what can be achieved in conventional lines, because of the fact that there are more degrees of freedom. In addition, metamaterial transmission lines can be designed to be electrically small, what makes them suitable for the synthesis of compact microwave circuits [6]. In the case of the resonant-type approach, the size of the line is determined by the size of the resonators and, for this reason, it is possible to obtain an important level of miniaturization (the use of electrically small resonators and the possibility to implement artificial lines with the required phase and impedance with a single unit cell is relevant for size reduction [7-9]).

In previous works by some of the authors, it was demonstrated that left-handed microstrip lines based on CSRRs were useful for the design of microwave components $[10,11]$. In this work, it is demonstrated that left-handed lines implemented in CPW technology and based on SRRs and other related topologies, such as the spiral resonator (SR), are also useful for the synthesis of compact microwave devices. Specifically, several power dividers have been designed, fabricated, and measured. Essentially, such power dividers consist on a $35.35 \Omega$ impedance inverter and two $50 \Omega$ output lines. It will be shown that the require impedance $(35.35 \Omega$ ) and phase $\left(90^{\circ}\right)$ of the inverter can be achieved with a single unit cell based on such resonators.

\section{TOPOLOGY AND CIRCUIT MODEL OF LEFT-HANDED LINES BASED ON SRRS}

As shown in Figure 1, a CPW can be loaded with magnetically coupled SRRs (etched in the back substrate side) and metallic strips placed between the central strip and the ground plane (acting thus as shunt connected inductances). A periodic structure consisting on a cascade of such unit cells can be considered, in a certain band above SRR's resonance, as a one-dimensional effective medium exhibiting left handed wave propagation (that is, with antiparallel phase and group velocities), and this has been interpreted as due to the negative effective permeability and permittivity of the line [4]. In this region, the phase constant is negative, as opposite to the positive phase constant of conventional lines. By using a single unit cell line, we can no longer consider the structure as an effective medium and, hence, we can not properly talk in terms of
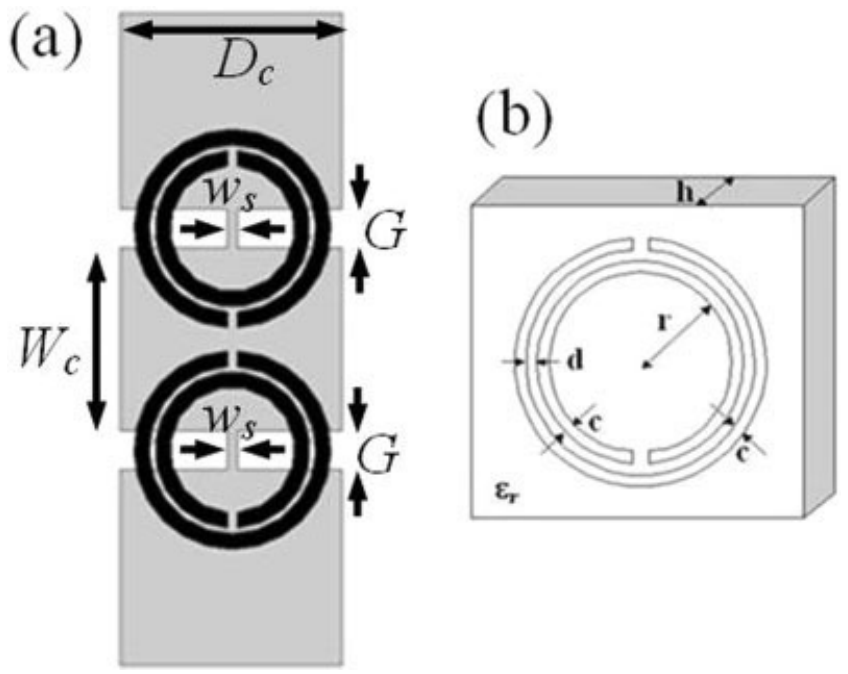

Figure 1 Layout of a left-handed cell in CPW technology (a), where the CPW (grey) is loaded with shunt inductances and magnetically coupled resonators on the other side of the substrate (black). Relevant dimensions for the SRR (b) 